\title{
Protesta, mercado e identidad en las celebraciones del Orgullo LGTB en España
}

\author{
Protest, market and identity in the LGTB Pride Celebrations in Spain
}

\author{
Begonya Enguix / benguix@uoc.edu \\ Orcid 0000-0002-5020-9019 \\ Universitat Oberta de Catalunya, España
}

\begin{abstract}
On the first Saturday of July, Madrid hosts the State LGTB (Lesbian, Gay, Bisexual and Transgender) Pride Demonstration with participants from LGTB activist groups, trade unions, political parties, NGOs and entrepreneurship. It involves around one million people and generates a profit of around 110 million euros. The participation of commercially sponsored floats along with the large influx of tourists that visit Madrid feed the discussions on the commercialization of the event and on the relationship between neoliberalism, identity and protest. These debates refer to the old tensions between critical activism and assimilationist activism. This article is based on a continued ethnographic fieldwork that combines participant observation, digital ethnography and in-depth interviews. We propose that the tensions between protest, activism, market and spectacle can be productive -as the Spanish LGTB movement has proved- and can create a new landscape of inclusive, hybrid and vindicating identity conceptions.
\end{abstract}

Key words: LGTB identities, marketization, new models for protest, hybridization.

Resumen: El primer sábado de julio Madrid acoge la Manifestación Estatal del Orgullo LGTB (Lesbianas, Gays, Bisexuales y personas Transgénero) en la que participan grupos activistas LGTB, partidos políticos y sindicatos, ONG y grupos empresariales. Asiste aproximadamente un millón de personas y genera unos beneficios que rondan los 110 millones de euros. Las carrozas esponsorizadas por empresas y la gran afluencia de turistas que recibe Madrid espolean los debates sobre la mercantilización de este evento y sobre la relación entre neoliberalismo, identidad y protesta. Dichos debates se inscriben en las tensiones entre el activismo identitario crítico y el asimilacionista. En este artículo, que se basa en un trabajo de campo etnográfico continuado que combina observación participante, etnografía digital y entrevistas en profundidad, proponemos que la tensión entre protesta, activismo, mercado y espectáculo puede ser productiva -como el movimiento LGTB ha demostrado en España- y puede generar concepciones identitarias inclusivas, híbridas y reivindicativas.

Palabras clave: identidades LGTB, mercantilización, nuevos modelos de protesta, hibridación. 


\section{Introducción $^{1}$}

Desde 1977, año de la primera manifestación por la liberación homosexual del Estado español, hasta la actualidad, el activismo LGTB en nuestro país ha sufrido importantes cambios, muchos de ellos como consecuencia de los avances legislativos obtenidos, como la legalización del matrimonio entre personas del mismo sexo en 2005. Esos cambios se pueden trazar a partir del estudio de las manifestaciones por la liberación homosexual/ Orgullo LGTB, pues éstas suponen la mayor y mejor expresión de visibilidad del colectivo LGTB, además del "acto activista LGTB por excelencia" (Toni Poveda²).

Las manifestaciones por el Orgullo LGTB tienen lugar cada año en más de 26 ciudades españolas como conmemoración de las revueltas de Stonewall (Nueva York, 1969). Entre ellas, destacan la manifestación Estatal por el Orgullo LGTB de Madrid (1.200.000 participantes en 2014, según los organizadores) y el Barcelona Pride (50.000 participantes en 2014, según los organizadores), por ser las más multitudinarias.

Si bien la manifestación catalana está organizada por la Asociación Catalana de Empresas para LGTB (ACEGAL) con la colaboración de asociaciones activistas, la manifestación de Madrid está organizada por el Colectivo de Lesbianas, Gays, Transexuales y Bisexuales de Madrid (COGAM) con el apoyo de la Federación Estatal de Lesbianas, Gays, Transexuales y Bisexuales (FELGTB), ambos colectivos activistas. Las manifestaciones de Barcelona y Madrid son el acto central de otros eventos festivos y culturales que se programan para las celebraciones del Orgullo. En Madrid, por ejemplo, "las fiestas del barrio las trabajan mano a mano COGAM y AEGAL (Asociación Empresarial para LGTB de Madrid), siendo esta última la encargada de la producción y programación de los escenarios" (OrgulloLGTB, 2016).

Una y otra son manifestaciones espectacularizadas (Debord, 2010) en las que cuerpos espectaculares desfilan junto a carrozas comerciales. No obstante, las diferencias en la organización se trasladan a su estructura, pues en Madrid existen dos secciones diferenciadas, una que se proclama claramente reivindicativa (en la cual participan algunas asociaciones LGTB del Estado -la mayoría amparadas por la FELGTB-, algunos colectivos críticos, ONG y partidos políticos y organizaciones sindicales), y otra más lúdico-festiva en donde participan carrozas de partidos políticos, asociaciones LGTB o esponsorizadas por negocios más o menos relacionados con la esfera LGTB.

1 Agradezco a Miguel Brox (COGAM) y a los activistas, políticos, empresarios y participantes entrevistados su colaboración en esta investigación.

2 Presidente de la FELGTB, entrevista 3 de febrero de 2010, sede FELGTB, Madrid. 
En contraste, el Pride Barcelona (llamado "desfile") es eminentemente comercial (Enguix, 2013) y no existe separación entre secciones.

La manifestación de Madrid es el objeto fundamental de análisis en este artículo por varias razones: se trata de la manifestación más importante del país (si la comparamos con cualquier otra manifestación pública, sea la del 1 de mayo o la del 8 de marzo) y es la mayor del Orgullo LGTB en Europa. Está convocada por la asociación LGTB más numerosa de España, COGAM, que tiene aproxidamente 400 socios. En colaboración con la Federación LGTB, saca a la calle a más de un millón de personas, según fuentes activistas que podrían estar un tanto exageradas. ${ }^{3}$ El desfase entre las cifras de socios y las de participantes plantea un interrogante sobre las estrategias utilizadas para conseguir esa enorme movilización.

En nuestra opinión, esas estrategias consisten fundamentalmente en la espectacularización del evento y la participación de carrozas esponsorizadas, ya que fue a partir de 1996 - primer año en que desfiló una carroza- cuando la manifestación empezó a crecer (Villaamil, 2004). Por tanto, Madrid es un caso ideal para analizar cómo se articulan las identidades (LGTB) con la protesta (merced al control activista del evento) y la comercialización.

Las manifestaciones por el Orgullo LGTB son consideradas como escaparate de las realidades LGTB y son objeto de múltiples críticas. Activistas, ciudadanos LGTB y otros actores sociales consideran que el modo de mostrar las identidades LGTB que allí se exhibe no representa la realidad LGTB contemporánea, ni es generalizable, ni puede ni debe serlo.

Así, es habitual encontrar en foros y comentarios a noticias en internet textos alusivos a este tema, en donde los participantes defienden el evento y la diversidad representacional que propicia o atacan unos "clichés" que consideran contraproducentes y/o contradictorios con la lucha por los derechos (Feijoo, 2013). Esta discusión sobre la representación está íntimamente relacionada con la crítica a la comercialización del evento.

Dicha comercialización es denunciada en manifestaciones alternativas -la organizada por la Comisión Unitaria 28 de junio en Barcelona, y la del Orgullo "Crítico", en Madrid, particularmente- que parten de posiciones queer y anticapitalistas, y tienen lugar en torno al 28 de junio. En 2014, la manifestación crítica de Madrid marchó bajo el lema "Orgullo es Decisión” y reunió a 500 personas. Desde la Asamblea “Orgullo Madrid 2014”, organizadora de la manifestación, señalan: "Salimos a las calles para gritar que el Orgullo es lucha, es decisión, es protesta, que el orgullo es nuestro, que no es un negocio, que no está en manos de políticos ni empresarios y que jamás lo estará” (García, 2014). En Barcelona, la manifestación del 28 de junio reunió a 5.000 personas.

3 Para el ala crítica del activismo, estas asociaciones son "oficialistas". 
No existen muchos textos de referencia sobre las manifestaciones del Orgullo LGTB, pues se trata de un campo incipiente de estudios, pero sí contamos con textos que abordan parcialmente cuestiones relacionadas con estas celebraciones. La mayor parte de los textos sobre el Orgullo lo estudian desde la perspectiva del turismo (Johnston, 2005; Murray, 2007; Puar et al., 2002; Binnie y Klesse, 2011).

La relación entre identidades LGTB y mercados ha sido analizada, entre otros, por Kates y Belk (2001), Johnston (2005), Sender (2004), Binnie y Skeggs (2004) y Chasin (2000a y b). Lesbianas y gays han sido con frecuencia representados como los pioneros de la gentrificación (Knopp, 1990; Lauria y Knopp, 1985) y como ciudadanos modelo para el consumo (Bell y Binnie, 2004). Mientras algunos autores critican la comercialización de las luchas LGTB (Bell y Binnie, 2004; Duggan, 2003), otros, como Hoggart y Woltersdorff (citados en Binnie, 2014: 245) ofrecen una perspectiva más ambivalente respecto a la relación entre la política LGTB y el neoliberalismo. Entre los efectos de esa relación se encuentra el de la exclusión de determinados sectores LGTB como consecuencia de la predominancia del consumo (Zukin, 2008; Peñaloza y Venkatesh, 2006; Binnie, 2014). La construcción de un nicho de mercado LGTB excluye generalmente a lesbianas y pobres, $y$, por tanto, está fuertemente interseccionada por el género y la clase.

Las fronteras entre la "normalización" (esgrimida como argumento por algunos informantes como objetivo final del Orgullo) y la "normativización" (reglamentación) son difusas: la construcción de las identidades LGTB para y por el consumo y la turistificación de las celebraciones urbanas conllevan para autores como Eribon (2000), Chasin (2000 a y b) y Hubbard (2001) la definición de un tipo de gay normativizado (asimilado, de clase media, blanco y con poder adquisitivo) que ha impuesto una homonormatividad que conlleva espirales de discriminación de gays, lesbianas, bisexuales y transexuales.

Este gay normativizado (o políticamente correcto) es una figura predominante en el Orgullo, y se contrapone con la primigenia liberación sexual. Markwell (2002) también incide en cómo las identidades LGTB pueden ser incluidas mediante el turismo y el marketing de las ciudades, pero cómo dicha inclusión está vinculada a una construcción muy estrecha de la identidad gay que excluye a quienes no se identifican con la esfera comercial. También hay que tener en cuenta que la conversión de gays y lesbianas en un nicho de mercado les constituye como ciudadanos/as en igualdad a través de prácticas consumistas (Chasin, 2000a). 
La articulación entre neoliberalismo, identidad y protesta se inscribe en un proceso más general (y generalizado) de transformación de las ciudades en espacios de consumo, ocio y entretenimiento. Fox-Gotham (2005 y 2012: 118) apunta que es conflictivo convertir las ciudades en espectáculos turísticos, porque las celebraciones y los espectáculos turísticos son una fuente de "caos" y "discontinuidad crónica".

Otros textos se centran en el Orgullo para elaborar el concepto de "vergüenza" (Johnston, 2007), para incidir en su carácter políticamente ambiguo, polémico y de resistencia ritualizada en tanto "festivales para el turismo" (Markwell y Waitt, 2009: 143) o para definirlo como una peculiar combinación de cultura popular y política que utiliza el humor (Lundberg, 2007). Blidon (2009: 2), por ejemplo, lo considera un fenómeno masivo de coming out urbano cuyo origen subversivo se ha perdido en lo festivo.

En España algunos autores que han trabajado sobre el activismo gay y la cultura queer tratan aspectos relacionados con lo que aquí se debate. ${ }^{4} \mathrm{Calvo}$ y Trujillo (2011) describen las tensiones entre el activismo mainstream y un activismo más radical, y Vidarte (2010) critica con fuerza los procesos de homonormatividad.

Este trabajo se basa en una investigación etnográfica (y, por tanto, cualitativa) iniciada en 2008. Sus unidades de análisis centrales son las celebraciones del Orgullo LGTB en España (y su relación con las instituciones públicas, la política y la empresa), el activismo LGTB en España y la conceptualización contemporánea de las identidades LGTB entendiéndose estos tres elementos como estrechamente relacionados. La investigación se centra fundamentalmente en Madrid por su importancia, aunque también se ha desarrollado en Barcelona, Sevilla y Valencia.

Desde 2008 se asistió a todas las manifestaciones del Orgullo LGTB de Madrid y, en función de sus fechas de celebración y la compatibilidad entre ellas, también a las manifestaciones ("oficialistas" y "críticas" si las había) de Barcelona, Sevilla y Valencia. ${ }^{5}$ El presente trabajo se basa sobre todo en la observación participante en estas manifestaciones y en las entrevistas en profundidad a informantes seleccionados, como técnicas fundamentales de investigación.

4 Véase, entre otros, VVAA (2012), Calvo y Pichardo (2011), Calvo y Trujillo (2011), Vidarte (2010), Mérida (2009), Herrero-Brasas (2007), Vergara et al. (2007) y Giorgi (2002).

5 En estas ciudades, la articulación y el equilibrio de fuerzas entre activistas, empresariado e instituciones públicas es diferente. 
En la observación participante en las manifestaciones, el registro, análisis e interpretación de los datos se ha llevado a cabo usando un diario de campo y registrando la actividad mediante procedimientos visuales (fotografías) y audiovisuales (video) propios de la antropología visual. Las fotografías tomadas durante las manifestaciones han sido, a su vez, utilizadas en las entrevistas como método de investigación (Collier y Collier, 1986).

La observación se complementa con la realización de entrevistas semiestructuradas en profundidad a informantes seleccionados mediante el método de la bola de nieve. Se ha entrevistado a un total de 44 personas: activistas LGTB, políticos, empresarios y participantes en las manifestaciones (manifestantes y personas que ponen carroza). ${ }^{6} \mathrm{El}$ acceso a los/as entrevistados/as ha sido facilitado por la trayectoria de investigación previa de la autora (Enguix, 1996 y 2014).

La etnografía digital ha sido también una fuente importante de información: desde 2009 se rastrean, clasifican y analizan webs de noticias, periódicos digitales y distintos blogs, foros y otras fuentes digitales detectadas mediante alertas de Google, para estudiar las noticias, comentarios y opiniones en torno a estos eventos.

Los datos digitales son hoy un elemento ineludible para el análisis de la realidad social. No obstante, seleccionarlos y acotarlos supone un reto en la investigación social, dado su número: en este trabajo se ha recurrido a las informaciones proporcionadas por los/as entrevistados/as y a elementos derivados de la observación para llevar a cabo esa tarea de selección.

Así, los datos obtenidos mediante procedimientos etnográficos presenciales han sido la base sobre la cual se han organizado e interpretado los datos digitales. El cruce entre etnografías presenciales y digitales, el análisis de contenido de bibliografía especializada y la realización de entrevistas a informantes situados en distintos posicionamientos sociales han favorecido una lectura difractiva (diffractive reading) (Mazzei, 2014) y la triangulación de los datos.

\section{Protesta y/o negocio}

Las celebraciones del Orgullo son un escenario privilegiado para analizar la tensión entre activismo y mercado (Sender, 2004; Chasin, 2000a), y la tensión entre protesta y fiesta (Debord, 2010). Dichas tensiones no son exclusivas ni se circunscriben a la manifestación del Orgullo sino que atraviesan también el barrio de Chueca, zona LGTB de Madrid. Durante la semana de la celebra-

6 Todos los entrevistados/as cuyas palabras aparecen en este trabajo han dado su consentimiento explícito a ser citados. 
ción del Orgullo, Madrid recibe alrededor de dos millones de turistas que dejan en la ciudad unos 110 millones de euros (Agencia EFE, 2012). Los locales de Chueca y el gremio de hosteleros de la ciudad parecen ser los grandes beneficiarios. AEGAL, la asociación de empresas para personas LGTB, es acusada de monopolizar los eventos mediante el control de los suministros a los bares y de las barras que se ponen en la calle (Lily, 2013). Pero es en la manifestación estatal donde se presentan con más claridad las tensiones entre protesta y negocio que son el foco de este trabajo.

Aunque la primera manifestación por la liberación homosexual de Madrid tuvo lugar en 1978, fue en los años noventa cuando siguiendo modelos extranjeros (fundamentalmente anglosajones) la manifestación fue girando hacia una creciente espectacularización algo carnavalesca. En 1996 participó en la marcha la primera carroza esponsorizada, la de la revista Shangay (dirigida por Alfonso Llopart). ${ }^{7}$ A partir de ese año, el número de asistentes se incrementó enormemente hasta llegar a más de un millón de participantes a partir de 2007 (año en que Madrid acogió la celebración del Europride), cifra que se ha ido manteniendo hasta el momento.

Aún así, los activistas han aspirado siempre a mantener el control del evento a diferencia de lo que ocurre en otros lugares del mundo como Nueva York y San Francisco, donde los eventos del Pride han sido "externalizados" y los realizan organizaciones creadas para tal efecto (NYCPride y SFPride) (San Francisco Lesbian Gay Bisexual Transgender Pride Celebration Committee, s/a). COGAM, como colectivo organizador, está a cargo de seleccionar las carrozas participantes y de clasificarlas de acuerdo con distintos criterios (OrgulloLGTB, 2016a).

No se acepta la participación de carrozas esponsorizadas por empresas manifiestamente contrarias a los colectivos LGTB. Las carrozas se clasifican en grupos con cuotas de participación diferenciales. En el primer grupo, COGAM, FELGTB y las carrozas de asociaciones LGTB desfilan primero, seguidas por sindicatos y secciones LGTB de partidos políticos (cuota A).

A partir de ese punto, el orden de participación se sortea entre los siguientes grupos: negocios LGTB que han participado con anterioridad (cuota A); negocios relacionados con el mundo LGTB que desfilan por primera vez (cuota B) y negocios no estrictamente relacionados con lo LGTB (cuota C), hasta llegar al límite de 40 carrozas impuesto por el Ayuntamiento de Madrid y la Delegación del Gobierno por motivos de seguridad.

7 Según Binnie y Klesse (2011: 169), el aumento de la esponsorización comercial de las celebraciones del Orgullo en Gran Bretaña se produjo también en los noventa. Asimismo, en Francia en esos años la marcha de París pasó de reunir a miles de personas, a reunir a cientos de miles con el pico del Europride en 1997 (Blidon, 2009: 6). 
Ser clasificado en uno u otro grupo no es baladí: afecta al orden que se ocupa en la marcha y también condiciona la cuota que las carrozas participantes pagan a COGAM (entre los 500 euros -cuota A- y los 6000 euros - cuota $\mathrm{C}-$ ) y que se destina a sufragar gastos de la organización y diversas campañas $\left(M . B^{8} x^{8}\right)$. COGAM intenta controlar la publicidad que se exhibe y en su normativa de carrozas establece que "las empresas cuya cuota de participación es reducida (cuotas A o B) no podrán llevar mas de un 10\% del espacio disponible en la carroza destinado a publicidad de otra marca o patrocinador que no sea la misma empresa".

Con el paso de los años, el número de carrozas ha ido variando: desde las 35 de 2010, a 18 en 2012, 13 en 2013 o 28 en 2014. Algunas decisiones de los colectivos activistas, como permitir la participación de empresas como Fiat, Google o Turismo de Fuerteventura, han sido muy discutidas:

Para mí la carroza también es un acto de visibilidad, es que el orgullo es visibilidad y es reivindicación de la dignidad, y es política. ¿Que es difícil? Evidentemente. ¿Que estamos vendidos al capital como la gente más radical dice? No es verdad, pero pisamos tierra $^{9}$ (T. Poveda).

La normativa de participación es estricta: la publicidad (excepto en cuotas C) está limitada y la carroza debe portar símbolos relativos a la comunidad LGTB y mostrar el eslogan oficial de la manifestación. Debe evitarse que parezcan carrozas de una cabalgata:

Se recuerda que las carrozas marchan en la Manifestación Estatal del Orgullo LGTB por lo que se exigirá que la decoración tenga referencias al tema (banderas arcoiris, triángulos rosas, etc.). Asimismo se exige llevar el logo del lema del Orgullo 2014 (OrgulloLGTB, 2016a).

Estas normas se suelen cumplir (al menos en lo que se refiere al eslogan, ya que en 2014 sólo dos carrozas no lo exhibían), aunque las carrozas, en general, parecen más discotecas ambulantes (con su música, sus bailarines, sus disfraces y su brillante colorido) que elementos reivindicativos.

La presencia de carrozas en las celebraciones del Orgullo muestra la centralidad del consumo en la cultura queer contemporánea (Valentine, 2002) y su rol como vertebrador de la identidad. ${ }^{10}$ Es una consecuencia de

8 Entrevista a Miguel Brox, socio de COGAM y ex responsable de carrozas, Madrid, 6 de noviembre de 2014.

9 Entrevista a Toni Poveda, ex presidente FELGTB, coordinador para el Orgullo en el momento de la entrevista, sede FELGTB, Madrid, 5 de febrero de 2013.

10 El mercantilismo de las identidades sexuales ha sido analizado, entre otros, por Hubbard (2001), Kates y Belk (2001), Valentine (2002), Rooke (2007), Holt y Griffin (2003), Taylor et al. (2002). Las traducciones son de la autora. 
la conversión de parte del colectivo LGTB (fundamentalmente los gays) en un nicho de mercado atractivo para muchos: Nike, por ejemplo, se mostró interesada en esponsorizar al primer atleta de alta competición que saliera del armario, de su mano. Las estrategias comerciales lgtbfriendly atraen a estos consumidores y con frecuencia son referidas como un elemento de "normalización”, como muestran estos comentarios a la noticia sobre Nike:

Convertir la salida del armario en un negocio podría restarle el plus de la superación, pero pensándolo bien creo que es lo más "rentable" para la normalización. Si hay algo que todo el mundo respeta es el dinero (Mañana, 2013).

Conclusión: Que hoy en día si eres un ídolo del deporte y eres gay es mucho mejor e incluso más rentable... todo sea con el objetivo de "normalizar" [traducción: imponer un pensamiento único y correctamente político y social] a los gays (Mañana, 2013).

Los gays, en mucha mayor medida que las lesbianas, los/as transexuales y los/as bisexuales, siguen siendo considerados como un segmento con un importante poder adquisitivo. Si bien este no es el lugar para debatir dicha cuestión, sí nos interesa destacar cómo el "segmento" se elabora en contraposición al "colectivo", de raíz más comunitaria e identitaria. Juan Pedro Tudela, fundador de la consultora Diversity Consulting, asesor del Ayuntamiento de Madrid y promotor de Fitur LGTB (el corner LGTB en la Feria de Turismo de Madrid, creado en 2011) afirma:

Las cadenas hoteleras no universalizan, normalizan, somos un segmento, no un colectivo... y hablo de gays y lesbianas, porque bisexuales y transexuales no son segmento... en el mundo occidental ya se han conseguido los derechos, las reivindicaciones activistas no tienen sentido y tampoco la forma como las llevan a cabo (tíos semidesnudos bailando encima de un bafle; o con tutú, tacones y tocado de monja) (entrevista realizada en Madrid, 3 de febrero de 2013). ${ }^{11}$

Juan Carlos Alonso, presidente del Comité Organizador del World Pride 2017 - que se celebrará en Madrid ese año- y secretario general de AEGAL, considera que esas afirmaciones son trasnochadas:

Creo que este discurso del "segmento gay tienen mucho dinero y es una oportunidad de negocio muy interesante" es un discurso muy simplista y trasnochado y te pongo un ejemplo muy claro. Hay un discurso que hay que empezar a cambiar. Los grandes estudios a nivel turismo, por ejemplo, de siempre, ponen al colectivo gay en lo que ellos llaman dink (double income no kids), vale, perfecto pero ¿qué ha ocurrido?: en España y en otros países las legislaciones avanzadas permiten matrimonios ya no sólo pareja, porque es mi novio o mi ligue o mi novia o mi ligue, con niños, que adoptan, entonces, esto ya cambia (entrevista realizada en la sede de AEGAL, Madrid, 4 de febrero de 2013).

11 Las transcripciones son literales y realizadas por la autora. Los posibles errores sintácticos son consecuencia de esa literalidad. 
Pero además, el ambiente festivo que se respira en la calle durante la marcha del Orgullo (que dura aproximadamente entre cuatro y cinco horas) y las numerosas actividades de todo tipo que se celebran en Madrid durante esos días tienen un enorme poder como reclamo turístico. La marca "Orgullo de Madrid” - estrategia no explicitada de city branding- ha convertido la ciudad en meca del turismo LGTB. ${ }^{12}$ La realidad LGTB como oportunidad de negocio (Chasin, 2000a; Peñaloza y Ventakesh, 2006) no escapa a los ojos de empresarios e instituciones públicas que al tiempo que dificultaban las negociaciones que cada año tenían lugar para la organización de la marcha, se asociaban con AEGAL para presentar (y obtener) la candidatura de Madrid para la celebración del World Pride en $2017 . .^{13}$

Así, el Orgullo de Madrid, y en particular los discursos en torno a su elemento más reivindicativo, la manifestación estatal, ejemplifica el giro de un capitalismo centrado en la produción, el trabajo y la coerción, al capitalismo de consumo basado en el ocio, la seducción del mercado y el espectáculo (FoxGotham, 2002; Ritzer, 2008), que convierte costumbres locales, rituales y festivales en atracciones para el consumo del turista y el mercado, al tiempo que son "herramienta de pacificación, de despolitización y masificación que 'distrae' y 'seduce' a la gente mediante los mecanismos del ocio, el consumo y el entretenimiento” (Fox-Gotham, 2002: 1737).

La contraposición de reivindicación y negocio, de protesta y mercado, puede ser paradójica pero no es necesariamente contradictoria: incluso considerando que el consumo se opone a los significados "verdaderos" que el Orgullo debería tener, como Fox-Gotham apunta y algunas narrativas sugieren, es indudable que el consumo gay también actúa como un elemento de refuerzo de la comunidad, generando modos de hacer y de mostrar que son identificables y en los cuales uno se puede reconocer.

Como Fiske (citado en Kates y Belk, 2001: 422) apuntó, la diversión y el placer no son incompatibles con los sentimientos políticos y de oposición al sistema; de hecho "el consumo conspicuo durante el día del Orgullo Gay y lésbico puede ser una actividad políticamente dudosa... pero la exhibición y muestra del poder de mercado puede de hecho conllevar una legitimación social de las comunidades gay y lesbiana” (Kates, 2002; Kates y Belk, 2001: 392).

Además, la separación entre activismo y negocio (o empresariado) debe ser problematizada y no sólo por lo expuesto hasta ahora: la mayoría de los 12 Gillbergy Adolfsson (2014) en su análisis de las webs de cinco ciudades nórdicas señalan cómo la diversidad ha devenido una estrategia de city branding.

13 El cambio político tras las elecciones municipales del 20 de mayo de 2015 ha hecho que esta situación de un giro radical. 
empresarios entrevistados que participan con carroza en la marcha dicen hacerlo por activismo. Aunque Alfonso Llopart, fundador de la revista Shangay y promotor de la primera carroza que desfiló en la manifestación, afirma:

Los empresarios no tienen conciencia política, yo tengo una opinión nefasta de los colectivos, nunca he militado. El $90 \%$ de gente que va al Orgullo va por la fiesta. $\mathrm{Si}$ desapareciera la primera parte de la mani no se perdería nada, si se perdiera la segunda volverían a ser cuatro. Yo propuse separarlas y COGAM se negó, porque sabe lo que perderían... Tras el matrimonio (2005), el Orgullo debería ser una celebración y otras demandas deberían buscar otros foros. Nadie va al Orgullo por reivindicación... ¿Orgullo mercantilizado?, pues sí (entrevista realizada en la sede de la revista Shangay, Madrid, 28 de febrero de 2013).

En idéntico sentido, Brox ${ }^{14}$ considera que existe una divisoria entre los empresarios LGTB de los años ochenta y los noventa, que eran activistas, y los actuales, a quienes se refiere con el término de "mercaderes" y cuya motivación activista desmiente.

La manifestación de Madrid, de origen identitario y reivindicativo, ha de sortear las pulsiones mercantiles representadas por su potencial turístico y por las carrozas. De ahí que haya quien en un alarde de exageración hable del "orgullo gay" (sic) como una actividad mercantil.

El activismo incide en su carácter reivindicativo al mantener el control de su organización y de las carrozas participantes (y recordando que deben mostrar el lema) y mediante la utilización de "marcadores activistas", como los numerosos banners y pancartas que predominan en la primera sección de la marcha y también en algunas carrozas (como la de 100 lesbianas visibles), los símbolos asociados a las realidades LGTB (banderas arcoiris y triángulo rosa), las consignas coreadas por el público, el uso de camisetas asociativas (Enguix, 2012a y 2012b), los voluntarios y voluntarias (de COGAM y FELGTB, principalmente) que monitorizan la marcha, y la existencia de una pancarta inicial con el eslogan de la marcha y la lectura de un manifiesto final. Estos elementos aspiran a recordar en todo momento que estamos ante un acto reivindicativo.

Aún así, en el seno del activismo son frecuentes los debates sobre los límites entre mercado y protesta que han dado lugar a una manifestación contestada pero que también les proporciona un poderoso argumento: el de la visibilidad, mediada por su capacidad de convocatoria.

14 Entrevista realizada en Madrid, el 6 de noviembre de 2014. 


\section{Hacia nuevos modelos de protesta}

En 1996 las carrozas esponsorizadas comenzaron a participar en la manifestación del Orgullo de Madrid. Aproximadamente cuatro o cinco años más tarde -las informaciones recibidas no son concluyentes-, se propuso convertir dicha manifestación en una Manifestación Estatal en la que participaran colectivos activistas procedentes de toda España. La espectacularidad creciente del evento, junto con un floreciente y liberal barrio de Chueca, convirtieron a la ciudad en meca del turismo LGTB en esas fechas.

En 2004 se creó AEGAL; en 2008: ACEGAL (Asociación Catalana de Empresas para LGTB); en 2009, ASEMGAL (Asociación Andaluza de Empresas para LGTB). No son las únicas asociaciones. Muchos empresarios eran y son activistas, otros no. Aunque en muchos casos el impulso para la creación de estas asociaciones ha surgido del activismo - para facilitar la interloculoción entre ambos ámbitos que colaboran estrechamente no sólo en el Orgullo sino en campañas de concienciación, información sobre el VIH y otras-, las tensiones entre unos y otros van en aumento.

Las intersecciones entre activismo (reivindicación y autenticidad del Orgullo), negocio (identidades versus nichos de mercado) y el city branding no permiten salidas fáciles e interrogan cómo construimos hoy las categorías identitarias y también cuáles son y serán las estrategias y alianzas presentes y futuras de los movimientos sociales de base identitaria.

Son diversas las preguntas que se plantean: ¿Pueden seguir presentándose en términos dicotómicos el activismo y la empresa? ¿Es posible conjugar la reivindicación con el mercado? En muchas de las entrevistas realizadas, sobre todo a activistas pero también a empresarios, se ha ensalzado el papel que los bares y discotecas LGTB -el ambiente- han tenido en la conformación de las "comunidades" LGTB y en proveer históricamente de lugares de reunión a dichas comunidades cuando la estigmatización social las condenaba al ostracismo. En palabras de Pedro Zerolo, activista gay y concejal del Ayuntamiento de Madrid, fallecido en 2015:

Nosotros fuimos sumando voluntades en Madrid y no sólo eran LGTB sino hombres y mujeres venidos de todo sitio y lugar... tan importantes han sido los colectivos en muchas partes de España como también los locales de reunión porque mientras luchábamos teníamos que socializar... Por cierto, empresarios gays y lesbianas que durante muchos años se partían la cara cuando en muchas ciudades no había ni siquiera colectivos y era el único lugar en el que podías socializar y en los cuales, en su apuesta empresarial había más de activismo que de rentabilidad. ¿Cómo no van a estar representados en el Orgullo esos empresarios gays, lesbianas, transexuales o bisexuales o heterosexuales que 
han destinado sus negocios, qué problema hay?, toda la gente que sale en el Orgullo incluidos los empresarios, sean como sean, las compañías grandes o pequeñas tienen que firmar los protocolos de adhesión por la igualdad, el reconocimiento en los convenios laborales de los derechos LGTB, yo creo que es importante (entrevista realizada en la sede del Ayuntamiento de Madrid, 13 de noviembre de 2011).

El activismo amparado en la FELGTB aboga así por la compleja articulación entre activismo y empresa y la justifica porque ha dado lugar a una fórmula de reivindicación, la de la manifestación en Madrid, cuyo éxito de participación y de público es único, dando con ello una gran visibilidad a la causa LGTB, al tiempo que equiparan la visibilidad al poder social (Toni Poveda, ex presidente FELGTB). ${ }^{15}$ Poveda afirma, además, que el modelo que se ha generado está sirviendo de ejemplo:

Yo he vivido la época en que éramos marginales y salíamos a la calle 40 personas. Nosotros hemos conseguido superarlo y muchos movimientos sociales están imitando el modelo del Orgullo. Quizá no sacan carrozas, pero están imitando el modelo del Orgullo. Desde que se celebran las manifestaciones del Orgullo, el 15M, los sindicatos, intentan dar color a las manifestaciones, que sean más atractivas. Yo no tengo ningún complejo además, pero incluso si vamos a la identidad y a la esencia de esta revolución, esta revolución empieza en un bar, en un bar, y a partir de ahí surge, pero no empieza en un bar por casualidad, porque era donde podían socializar las personas LGTB de una manera clandestina, ilegal o como queramos llamarle, pero empieza ahí (entrevista realizada en la sede de la FELGTB, Madrid, 5 de febrero de 2013).

Muchos líderes activistas entrevistados destacan, además, que es un valor añadido que una empresa participe en el Orgullo, porque con ello se muestra próxima a la reivindicación LGTB.

Los discursos contrarios a esta articulación -individuales o en las manifestaciones críticas- obedecen a una lógica de autenticidad que contrapone la lógica reivindicativa con la lógica comercial. Beben de una tradición en la cual la militancia era considerada contradictoria con la asistencia a los bares y otros lugares del ambiente, que eran considerados como un gueto y no como espacios de liberación (Eribon, 2000). ${ }^{16}$ Desde el activismo crítico se aspira a que la lucha por el derecho a la propia sexualidad no se diferencie de la lucha por el derecho a la vivienda o a la educación (E. Rodríguez, portavoz FAGC).

En una línea similar a Rodríguez, también desde el activismo crítico, Gracia Trujillo afirma:

15 Entrevista realizada en la sede de la FELGTB, Madrid, el 5 de febrero de 2013.

16 Para más información sobre la contraposición entre militancia y ambiente, véase Enguix (1996) y Calvo y Trujillo (2011). 
¿Por qué tienen que participar empresas en una manifestación? Yo voy a muchas otras, como el $1^{\circ}$ de Mayo o el 8 de Marzo y no hay empresas sacando tajada de nada. Me parece un ejemplo de la invasión de espacios de protesta por parte del neoliberalismo y del "te tolero porque consumes y mientras consumas" que es algo, precisamente, intolerable. Los derechos de lesbianas, gays, trans, bisexuales, intersexuales, queers no son un negocio, como no lo son los de cualquier grupo discriminado. Como nos descuidemos, en este contexto de privatizaciones del espacio público, nos privatizan la manifestación!... Hoy en día sigue siendo necesario que se nos vea, pero yo creo que la visibilidad tiene que ser algo estratégico sobre lo que cada uno a decide (Gracia Trujillo, profesora de Sociología y activista queer, comunicación personal, febrero de 2013).

Entre ambas posiciones, que aparecen como incompatibles, cabe preguntarse si la mercantilización y la publicidad son los únicos medios hoy para conseguir visibilidad, puesto que la visibilidad se dibuja como un elemento clave en relación con un elevado índice de asistencia:

Para mí el objetivo es la celebración de una fecha reivindicativa para continuar visibilizando nuestra reivindicación. Si la fórmula de Orgullo que buscamos no sale en ningún sitio pues estaríamos celebrando que en el año 69 pasó aquello de Stonewall y ya está, pues para eso hago una fiesta en mi casa, no sé. La colaboración con el empresariado, hoy por hoy, la veo imprescindible... en este ámbito el purismo no nos lleva a ningún sitio porque si hemos llegado donde hemos llegado es porque hemos asumido muchas contradicciones también, se han quedado muchas cosas por el camino y al final pues se negocia y ahora continuamos reivindicando lo que nos falta... a alguna gente le encanta ser marginal, pero al final, en mi experiencia, después de tantos años, lo que la gente viene a pedirnos es vivir en libertad, no ser marginal (entrevista a José de Lamo, presidente del Col.lectiu Lambda en el momento de la entrevista [enero 2013] y posteriormente secretario de Organización de la FELGTB. Traducida del catalán por la autora).

La espectacularización que ha fagocitado las manifestaciones LGTB en mucha mayor medida que otras manifestaciones político-reivindicativas, da lugar a un modelo específico con gran éxito de participación y público: la "manifiesta", término creado por Ruth Toledano. No hay más que comparar el número de asistentes a las manifestaciones "críticas" y "oficialistas". Sin duda, en ello tiene que ver la constitución de gays y lesbianas (sobre todo gays) como nicho de mercado y la tradición de colaboración entre activismo y empresa a la que se ha aludido. Pero esa colaboración debe remitir, según los más críticos. Eugeni Rodríguez, portavoz del Front d'Alliberament Gai de Catalunya (FAGC) que organiza, con otros colectivos, el orgullo "crítico" de Barcelona, afirma: 
¿El 8 de Marzo lo convoca la Evax? No, y les hace a las mujeres un servicio impresionante. Me da igual que convoque una empresa que da servicios a los gays. Son marcas... los derechos los hacen las personas que luchan por ellos, el tejido asociativo son las organizaciones, no una empresa (entrevista realizada en la sede del FAGC, Barcelona, 5 de mayo de 2014).

\section{Identidades híbridas: Consideraciones finales}

Este caso expone la difícil articulación entre identidad, protesta y mercado. Dicha tensión se inscribe en las distintas estrategias reivindicativas adoptadas por los movimientos sociales de raíz identitaria, que oscilan entre la posición asimilacionista -de "normalización del hecho sexual" (Coll-Planas, 2008: 51) apelando a la identidad/identificación- y las posiciones radicales, liberacionistas o de "transformación social" (Coll-Planas, 2008: 51) que propugnan la lucha por el reconocimiento a la diferencia (Calvo y Trujillo, 2011; Arditi y Hequembourg, 1999; Bernstein, 1997; Rimmerman, 2008). ${ }^{17}$

La tensión entre identidad y diferencia es intrínseca a estos movimientos sociales (Bourdieu, 2005: 145), siendo el movimiento LGTB la quintaesencia de los movimientos identitarios por su implicación en la transformación cultural de la sociedad, la adquisición de derechos y sus consecuencias identitarias. Las contradicciones, tensiones y dificultades de los movimientos identitarios están relacionadas con lo que Ghaziani (2008: 8) llama "falso universalismo", la creencia en la homogeneidad identitaria y social, y este caso no es una excepción.

El mercado y las relaciones con la empresa ejemplifican la tensión entre el activismo "oficialista"/asimilacionista (FELGTB y grupos satélite) y el activismo crítico y anticapitalista/radical (FAGC, Brot Bord, Asamblea Transmaricabollo y otros). Las identidades mercantilizadas -y asimilablesse construyen como (falsos) universales y aspiran a ser universalizadas. Se construyen, además, como opuestas e incompatibles con la protesta y la reivindicación.

Es urgente revisar la universalización identitaria y la oposición entre mercado y protesta, porque ambos procesos esencializan y otorgan carácter de "realidad" a las fronteras entre estilos de vida, nichos de mercado, reivindicación e identidades (dibujándolos de manera excluyente) cuando hoy esos límites son difusos, movibles, poco estables y sumamente problemáticos.

17 Según Calvo y Trujillo (2011: 565), la sección “institucionalizada” del movimiento LGTB, la mayor y más visible, ha abrazado modos de reivindicación "desexualizados", apelando a nociones como los derechos humanos, la ciudadanía e incluso la nación. 
Debemos incorporar a nuestros análisis nuevos modelos identitarios que redefinan esos límites y consideren tanto las prácticas, los deseos y las experiencias sexuales como los estilos de vida vehiculados por el mercado o por el enfrentamiento a él, así como su expresión política. En ello inciden todos los líderes activistas entrevistados al apelar a la diversidad y a la integración de todas las perspectivas.

La legislación española respecto a la realidad LGTB es más avanzada que nunca y es indudable que las estrategias asimilacionistas adoptadas por el activismo español (e internacional) han dado sus frutos. ${ }^{18}$ Buena parte de los cambios sociales acaecidos se deben a las movilizaciones LGTB (Calvo y Trujillo, 2011), pero también a un viraje del radicalismo a la moderación que se inició en los años ochenta (Calvo y Trujillo, 2011: 575).

En el contexto de crisis actual, no obstante, esas victorias pueden ser consideradas como “tenues y frágiles" (D’ Emilio, 1993: 467). Por ello, hay que recordar que gays, lesbianas, transexuales y bisexuales son seres históricos cuya identidad sólo fue posible en relación con las relaciones capitalistas (D’ Emilio, 1993: 468) y gracias a la nueva organización del trabajo, de la familia y a la desvinculación entre sexualidad y procreación que el capitalismo forjó.

La estructura del capitalismo hizo posible la emergencia de una identidad gay y la creación de comunidades gays urbanas (D’ Emilio, 1993: 473) y es esa misma estructura la que hoy puede fagocitar y diluir tanto la identidad como su expresión política. Si bien la globalización capitalista supone en muchos lugares la adopción de nuevos modelos de protesta -mucho más mercantilizados que el español- también favorece la emancipación en lugares donde de otro modo ésta no sería posible, como Polonia (Binnie y Klesse, 2011: 165).

Criminalizar al empresariado parece un camino sin salida, no sólo por su papel histórico y reconocido, sino porque gracias a las carrozas la manifestación consigue sacar a la calle a gran cantidad de personas. También lo es contraponer la protesta y el mercado o diluir el contenido reivindicativo en lo empresarial, como ha ocurrido en tantos lugares. Oponer protesta y mercado es estéril.

Los límites de las identidades, las reivindicaciones y los negocios identitarios (identity-based) deben ser redefinidos de forma no excluyente ni exclusiva, sino inclusiva. La propia definición de la categoría LGTB es hoy híbrida (Hall, 1992; García-Canclini, 2001). La práctica sexual no sólo produce identidades - prácticas, deseos, experiencias-, sino cuerpos de consumo, para el consumo y cuerpos de consumidores.

18 Baird (citado en Binnie y Klesse, 2011) vincula las reformas legales con la promoción turística de las ciudades como "gayfriendly". 
El reto para el activismo LGTB (que sigue actuando como cohesionador y portavoz de las identidades LGTB y como depositario de la reivindicación identitaria) consiste en generar discursos integradores sin pérdida de la "autenticidad" reivindicativa que puede quedar oculta, porque deslumbrados por las carrozas no veamos las pancartas; consiste en vencer aquellos intereses que aspiran a despolitizar el evento negando la relevancia de quienes marchan para destacar solamente a quienes bailan. ${ }^{19}$

$\mathrm{El}$ reto es mantenerse como depositarias de los discursos reivindicativos LGTB en un contexto donde muchos consideran que ya no tienen sentido, dados los avances conseguidos, mientras muchos otros consideran que "queda mucho por hacer en pro de la igualdad efectiva”. El reto es posicionarse en este nuevo y complejo contexto, manteniéndose como asociaciones políticas además de asistenciales (ONG). La celebración del Orgullo -como acto de visibilidad y protesta - es el evento central que otorga legitimidad política a las asociaciones LGTB y, por tanto, su evolución influirá en nuestra definición de las identidades, el activismo y la acción política LGTB.

Este caso revela la urgencia de interrogar cómo construimos las identidades hoy y problematizar la idea de que el mercado conlleva su desaparición. Sabemos que las identidades son múltiples, inacabadas y contextualmente construidas sociohistóricamente (Foucault, 1984; Butler, 1990; Enguix, 2000), que gays y lesbianas no son "una minoría social fija compuesta por un cierto porcentaje de personas y duradera y estable en el tiempo" (D' Emilio, 1993: 473).

Sabemos que las múltiples expresiones e identidades forjadas sociohistóricamente no pueden ser entendidas como estructuras fijas y estancas ni les son de aplicabilidad niveles distintos de "autenticidad". Pero aún así, seguimos construyendo modelos dicotómicos y excluyentes. Hoy es posible pensar identidades híbridas que conjuguen elementos relacionados con el mercado sin que los grupos queden constituidos única y exclusivamente como nichos de mercado y para el mercado. Necesitamos nuevos modelos identitarios que afronten esta complejidad a partir de la modularidad y la transformación.

Movilización, visibilidad y poder están íntimamente ligados hoy con la lógica capitalista y con la acción colectiva y política. Las tensiones y el conflicto son inherentes a las marchas LGTB y son cruciales para definir tanto las identidades como las estrategias del movimiento (Ghaziani, 2008). Los conflictos y la disidencia pueden ser productivos, producir nuevas formas de

19 Comparando las noticias publicadas en prensa sobre la manifestación en 2009 y 2010 se observó una fuerte tendencia hacia la despolitización y desideologización del evento (Enguix, 2013). 
organización y de protesta. El caso español es peculiar en un contexto global en el que las celebraciones del Orgullo o bien están totalmente mercantilizadas, o bien están prohibidas: Madrid tiene la oportunidad de encontrar el camino intermedio, un modelo híbrido, que supere los antiguos esencialismos que ya no son útiles. ${ }^{20}$

El reto es redefinir las identidades y la protesta de modo que la segunda no coarte las primeras ni desaparezca al tiempo que se establecen alianzas claras con empresariado e instituciones para no convertir lo identitario en un mero espectáculo sin contenido.

\section{Referencias}

Agencia EFE (2012) "Los organizadores del Orgullo Gay denuncian falta de apoyo del ayuntamiento de Madrid”. Disponible en: http://www.publico.es/espana/430750/ los-organizadores-del-orgullo-gay-denuncian-falta-de-apoyo-del-ayuntamiento-demadrid [20 octubre 2014].

Arditi, Jorge y Hequembourg, Amy (1999), "Modificaciones parciales: discursos de resistencia de gays y lesbianas en Estados Unidos”, en Política y Sociedad, vol. 30, España: Universidad Complutense de Madrid.

Bell, D. y Binnie, John (2004), “Authenticating Queer Space: Citizenship, Urbanism and Governance”, en Urban Studies, vol. 41, núm. 9, Inglaterra y Estados Unidos: Sage.

Bernstein, Mary (1997), "Celebration and suppression: The strategic uses of identity by the lesbian and gay movement”, en The American Journal of Sociology, vol. 103, núm. 3, Estados Unidos: The University of Chicago.

Blidon, Marianne (2009), "La Gay Pride entre subversion et banalisation”, en Espace, Populations, Sociétés, vol. 2, Francia: Université de Lille.

Binnie, Jon y Skeggs, Beverley (2004), "Cosmopolitan knowledge and the production and consumption of sexualized space: Manchester's gay village”, en The Sociological Review, vol. 52, núm. 1, Inglaterra: Goldsmiths University-The Sociological Review Publication Limited.

Binnie, Jon y Klesse, Christian (2011), "Because It Was a Bit Like Going to an Adventure Park': The Politics of Hospitality in Transnational Lesbian, Gay, Bisexual, Transgender and Queer Activist Networks", en Tourist Studies, vol. 11, núm. 2, Inglaterra y Estados Unidos: Sage.

Binnie, Jon (2014), "Neoliberalism, Class, Gender and Lesbian, Gay, Bisexual, Transgender and Queer Politics in Poland", en International Journal of Politics, Culture and Society, vol. 27, núm. 2, Estados Unidos: Springer.

Bourdieu, Pierre (2005), La dominación masculina, España: Anagrama.

Butler, J. (1990), Gender trouble, Estados Unidos: Routledge.

20 Markwell y Waitt (2009: 163) muestran cómo en Australia conviven distintos modelos en la celebración del Orgullo, pero ninguno combina la reivindicación y el negocio, que se dan como modelos excluyentes y exclusivos. 
Calvo, Kerman y Pichardo, J. I. (2011), “Sexualities Transformed? Inside Visions of Sexual, Social and Political Change in Spain”, en Sexualities, vol. 14, núm. 5, Inglaterra y Estados Unidos: Sage.

Calvo, Kerman y Trujillo, G. (2011), "Fighting for Love Rights: Claims and Strategies of the LGTB Movement in Spain”, en Sexualities, vol. 14, núm. 5, Inglaterra y Estados Unidos: Sage.

Chasin, Alexandra (2000a), Selling Out. The Gay and Lesbian Movement goes to the Market, Estados Unidos: Palgrave.

Chasin, Alexandra (2000b), "Interpenetrations: A Cultural Study of the Relationship between the Gay/Lesbian Niche Market and the Gay/Lesbian Political Movement", en Cultural Critique, núm. 44, Estados Unidos: University of Minnesota Press.

Collier, John Jr. y Collier, Malcolm (1986), Visual Anthropology: Photography as a Research Method, Estados Unidos: University of New Mexico Press.

Coll-Planas, Gerard (2008), "Homosexuals, bolleres i rarets: posicions polítiques en el moviment lèsbic i gai”, en Athenea digital, núm. 14, España: Universitat Autònoma de Barcelona.

Debord, Guy (2010), Society of the Spectacle, Estados Unidos: Black and Red.

D’Emilio, John (1993), "Capitalism and Gay Identity”, en The Lesbian and Gay Studies Reader, Estados Unidos: Routledge.

Duggan, Lisa (2003), The Twilight of Equality? Neoliberalism, Cultural Poitics and the Attack on Democracy, Estados Unidos: Beacon Press.

Enguix, Begonya (1996), Poder y Deseo. Aproximación al Análisis de la Homosexualidad Masculina, España: Alfons el Magnànim.

Enguix, Begonya (2000), "Sexualidad e Identidades", en Gazeta de Antropología, núm. 16, art. 4. Disponible en: http://www.ugr.es/ pwlac/G16_04Begona_Enguix_Grau.pdf [11 julio 2015].

Enguix, Begonya (2009a), "Identities, Sexualities and Commemorations: Pride Parades, Public Space and Sexual Dissidence”, en Anthropological Notebooks, vol. XV, núm. 2, Eslovenia: Slovenian Anthropological Society.

Enguix, Begonya (2009b), "Espacios y disidencias. El orgullo LGTB”, en Quaderns-e (Barcelona), núm. 14, España: Institut Català d’Antropologia.

Enguix, Begonya (2012a), "Cuerpos, Camisetas e Identidades como Estrategias de Protesta”, en Tejerina, Benjamín y Perugorría, Ignacia [eds.], Global Movements, National Grievances. Mobilizing for 'Real Democracy' and Social Justice, España: Servicio Editorial de la Universidad del País Vasco.

Enguix, Begonya (2012b), “Cuerpos y Protesta: Estrategias Corporales en la Acción Colectiva”, en Revista Brasilera de Sociologia da Emoção, vol. 11, núm. 33, Brasil: Universidade Federal da Paraíba.

Enguix, Begonya (2013), "Sexual Politics, Pride, and Media Mediation in Spain”, en Observatorio OBS*, vol. 7, núm. 2). Disponible en: http://obs.obercom.pt [20 de julio de 2014].

Enguix, Begonya (2014), "Negotiating the Field: Rethinking Ethnographic Authority, Experience and the Frontiers of Research", en Qualitative Research, vol. 14, núm. 1, Inglaterra y Estados Unidos: Sage.

Eribon, Didier (2000), Identidades. Reflexiones sobre la Cuestión Gay, España: Bellaterra. 
Convergencia Revista de Ciencias Sociales, núm. 73, 2017, Universidad Autónoma del Estado de México

F., Diego (2013), "Un concurso de fotos sobre el Orgullo Gay, contra la extravagancia". Disponible en: http://www.cromosomax.com/22400-un-concurso-de-fotos-sobreel-orgullo-gay-contra-la-extravagancia [6 julio 2013 y 15 enero 2014].

Feijoo, Diego (2013), "Un concurso de fotos sobre el Orgullo Gay, contra la extravagancia”. Disponible en: http://www.cromosomax.com/22400-un-concurso-de-fotos-sobre-elorgullo-gay-contra-la-extravagancia [15 enero 2014].

Foucault, Michel (1984), Historia de la Sexualidad (I). La Voluntad de Saber, España: Siglo XXI.

Fox-Gotham, Kevin (2002), "Marketing Mardi Gras: Commodification, Spectacle and the Political Economy of Tourism in New Orleans”, en Urban Studies, vol. 39, núm. 10, Inglaterra y Estados Unidos: Sage.

Fox-Gotham, Kevin (2005), "Theorizing urban spectacles: Festivals, tourism and the transformation of urban space”, en City, vol. 9, núm. 2, Inglaterra: Taylor and Francis Group.

Fox-Gotham, Kevin (2012), “Theorizing Carnival. Mardi Gras as perceived, conceived and lived space", en Brown, Jerome y Langman, Lauren [eds.], Alienation and the Carnivalization of Society, Estados Unidos: Routledge.

García-Canclini, Néstor (2001), Culturas híbridas. Estrategias para entrar y salir de la modernidad, Estados Unidos: Paidós.

García, Daniel (2014), “Orgullo es decisión, la manifestación del 28J en Madrid”. Disponible en: http://noticias.universogay.com/orgullo-es-decision-la-manifestacion-del-28j-enmadrid_27062014.html [17 agosto 2014].

Ghaziani, Amin (2008), The Dividends of Dissent. How Culture and Conflict work in Lesbian and Gay Marches on Washington, Estados Unidos: The University of Chicago Press.

Gillberg, Nanna y Adolfsson, Petra (2014), Proud to Be Pride: A Discourse Analysis of the Presentation of Diversity on City Websites, Suecia: Gothenburg Research Institute.

Giorgi, Gabriel (2002), "Madrid en Tránsito: Travellers, Visibility and Gay Identity”, en GLQ, Journal of Lesbian and Gay Studies, vol. 8, núm. 1/2, Inglaterra: Duke University Press.

Hall, S. (1992), “The question of cultural identity”, en Hall, S., Held, D. y McGrew, T., [eds.], Modernity and its future, Inglaterra: Polity.

Herrero Brasas, Juan A. [ed.] (2007), Primera Plana. La Construcción de una cultura queer en España. España: Egales.

Holt, Martin y Griffin, Christine (2003), "Being Gay, Being Straight and Being Yourself: local and global Reflections on Identity, Authenticity and the Lesbian and Gay Scene", en European Journal of Cultural Studies, vol. 6, núm. 3, Inglaterra y Estados Unidos: Sage.

Hubbard, Phil (2001), “Sex Zones: Intimacy, Citizenship and Public Space”, en Sexualities, vol. 4, núm. 1, Inglaterra y Estados Unidos: Sage.

Johnston, Linda (2005), Queering Tourism. Paradoxical Performances at Gay Pride Parades, New York: Routledge.

Johnston, Linda (2007), “Mobilizing pride/shame: lesbians, tourism and parades”, en Social \& Cultural Geography, vol. 8, núm. 1, Inglaterra: Taylor and Francis Group

Kates, Steven M. y Belk, Russell (2001), “The Meanings of Lesbian and Gay Pride Day: Resistance through Consumption and Resistance to Consumption”, en Journal of Contemporary Ethnography, vol. 30, núm. 4, Inglaterra y Estados Unidos: Sage. 
Kates, Steven M. (2002), “The Protean Quality of Subcultural Consumption: An Ethnographic Account of Gay Consumers", en Journal of Consumer Research, vol. 29, núm. 3, Estados Unidos: Journal of Consumer Research.

Knopp, L. (1990), "Some Theoretical Implications of Gay Involvement in an Urban Land Market”, en Political Geography Quarterly, vol. 9, núm. 4, Holanda: Elsevier.

Lauria, M. y Knopp, L. (1985), "Towards an Analysis of the Role of Gay Communities in the Urban Renaissance”, en Urban Geography, vol. 6, núm. 2, Inglaterra: Taylor and Francis Group.

Lily, Shangay (2013), "MADO: Orgullo Gaypitalista”. Disponible en: http://blogs.publico. es/shangaylily/2013/07/06/mado-orgullo-gaypitalista/ [26 julio 2014].

Lundberg, Anna (2007), "Queeringlaughter in the Stockholm Pride Parade”, en International Review of Social History, vol. 52, Inglaterra: Cambridge University Press.

Mañana, Carmen (2013), "Se busca deportista gay para anuncio multimillonario", en El País. Disponible en: http://elpais.com/elpais/2013/04/30/gente/1367340026_100946. html [15 junio 2014].

Markwell, K. (2002), "Mardi Gras Tourism and the Construction of Sydney as an International Gay and Lesbian City", en GLQ: A Journal of Lesbian and Gay Studies, vol. 8, núm. 1/2, Inglaterra: Duke University Press.

Markwell, Kevin y Waitt, Gordon (2009), "Festivals, space and sexuality: Gay Pride in Australia”, en Tourism Geographies, vol. 11, núm. 2, Inglaterra: Taylor and Francis Group.

Mazzei, L. (2014), "Beyond an Easy Sense: A Diffractive Analysis”, en Qualitative Inquiry, vol. 20, núm. 6, Inglaterra y Estados Unidos: Sage.

Mérida-Jimenez, Rafael, M. [ed.] (2009), Manifiestos Gays, Lesbianos y Queer. Testimonios de una Lucha (1969-1994), España: Icaria.

Murray, David (2007), “The Civilized Homosexual: Travel Talk and the Project of Gay Identity”, en Sexualities, vol. 10, núm. 1, Inglaterra y Estados Unidos: Sage.

New York City Pride (s/a), Heritage of Pride Inc. Disponible en: https://www.nycpride. org/ [27 octubre 2014].

OgulloLGTB (2016), Información oficial del Orgullo LGBT de Madrid 2016 organizado por COGAM y FELGTB. Disponible en: http://orgullolgbt.org/preguntas-frecuentesorgull/ [17 agosto 2014].

OrgulloLGTB (2016a), “Carrozas”. Disponible en: http://orgullolgbt.org/ manifestacion-2/orgullo-es-cultura/ [10 septiembre 2014].

Peñaloza, Lisa y Venkatesh, Alladi (2006), "Further evolving the new dominant logic of marketing: from services to the social construction of markets", en Marketing Theory, vol. 6, núm. 3, Inglaterra y Estados Unidos: Sage.

Puar, Jasbir Kaur [ed.] (2002), Queer Tourism, Geographies of Globalization, GLQ a Journal of Lesbian and Gay Studies, vol. 8, núm. 1-2, Estados Unidos: Duke University Press.

Rimmerman, Craig A. (2008), The Lesbian and Gay Movements. Assimilation or Liberation?, Estados Unidos: Westview.

Ritzer, George (2008), La McDonalización de la Sociedad. Un análisis de la racionalización en la vida cotidiana, España: Ariel Sociedad Económica.

Rooke, Alison (2007), "Navigating Embodied Lesbian Cultural Space: Toward a Lesbian habitus”, en Space and Culture, vol. 10, núm. 2, Inglaterra y Estados Unidos: Sage. 
Sender, Katherine (2004), Business, not Politics. The Making of the Gay Market, Estados Unidos: Columbia University Press.

Taylor, Verta et al. (2002), "From the Bowery to the Castro: Communities, Identities and Movements", en Richardson, Diane y Seidman, Steven [eds.], Handbook of Lesbian and Gay Studies, Inglaterra: Sage.

Valentine, Gill (2002), "Queer Bodies and the Production of space”, en Richardson, Diane y Seidman, Steven [eds.], Handbook of Lesbian and Gay Studies, Inglaterra: Sage.

VVAA (2012), El Orgullo es Nuestro, Madrid: Libros de Diagonal.

Vergara, Elena et al. (2007), El camino hacia la igualdad. 30 Años de lucha por los derechos $L G T B$ en el Estado Español, España: COGAM (Colectivo de Lesbianas, Gays, Transexuales y Bisexuales de Madrid).

Vidarte, Paco (2010), Ética Marica. Proclamas Libertarias para una Militancia LGBTQ, España: Egales.

Villaamil, Fernando (2004), La Transformación de la Identidad Gay en España, España: Libros de la Catarata.

Zukin, Sharon (2008), "Consuming authenticity”, en Cultural Studies, vol. 22, núm. 5, Inglaterra: Taylor and Francis.

Begonya Enguix. Doctora en Antropología Social y Cultural (URV) y licenciada en publicidad (UOC). Es profesora agregada de los Estudios de Artes y Humanidades de la Universitat Oberta de Catalunya y directora en esta institución del Grado de Antropología y Evolución Humana URVUOC. Principales líneas de investigación: género, cuerpo, sexualidades, identidad, antropología urbana, metodologías de investigación y antropología de los media. Publicaciones recientes: "Negotiating the Field: Rethinking Ethnographic Authority, Experience and the Frontiers of Research", en Qualitative Research, Londres y Nueva York: Sage (2014); Rethinking Romantic Love: Discussions, Imaginaries and Practices, Cambridge: Cambridge Scholars Publishing (2015), Enguix, B. y Roca, J. [eds.]; "Activism and Digital Practices in (for) the Construction of a Spanish LGTB Sphere”, en Dados, Revista de Ciencias Sociais, Río de Janeiro: Universidade do estado do Rio de Janeiro (2016, en prensa).

Recepción: 11 de julio de 2015.

Aprobación: 21 de octubre de 2016. 